\title{
Subject Index Volume 4
}

ABCC8

clinical and functional characterization of the Pro1198Leu ABCC8 gene mutation associated with permanent neonatal diabetes mellitus, Takagi 269-273

adipocyte fatty acid binding protein

obesity as the common soil of non-alcoholic fatty liver disease and diabetes: role of adipokines (Review Article), Hui $413-425$ adipokines

glypican-4 is a new comer of adipokines working as insulin sensitizer, Tamori 250-251

adiponectin

obesity as the common soil of non-alcoholic fatty liver disease and diabetes: role of adipokines (Review Article), Hui 413-425

serum adiponectin levels predict the risk of coronary heart disease in Japanese patients with type 2 diabetes, Obata 475-482

adverse drug reaction

three ileus cases associated with the use of DPP-4 inhibitors in diabetic patients (Case Report), Kanasaki 676

albuminuria

comparison of effects of cilnidipine and azelnidipine on blood pressure, heart rate and albuminuria in type 2 diabetics with hypertension: a pilot study, Abe 202-205

comparison of spironolactone and trichlormethiazide as add-on therapy to renin-angiotensin blockade for reduction of albuminuria in diabetic patients, Hase 316-319

aldosterone blockers

comparison of spironolactone and trichlormethiazide as add-on therapy to renin-angiotensin blockade for reduction of albuminuria in diabetic patients, Hase 316-319

Alzheimer's disease

insulin resistance in the brain: a new therapeutic target for Alzheimer's disease (Commentary), Umegaki 150-151

new therapeutic strategy for Alzheimer's disease using antidiabetes agents (Commentary), Yokono 152-153

angiotensin-converting enzyme gene

angiotensin-converting enzyme gene variants interact with the renin-angiotensin system pathway to confer risk and protection against type 2 diabetic retinopathy (Letter to the Editor), Singh Cheema 103-104

angiotensin-converting enzyme inhibitor

combination therapy with an angiotensin-convertingenzyme inhibitor and an angiotensin II receptor antagonist ameliorates microinflammation and oxidative stress in patients with diabetic nephropathy, Nakamura 195-201

angiotensin receptor blocker

combination therapy with an angiotensin-convertingenzyme inhibitor and an angiotensin II receptor antagonist ameliorates microinflammation and oxidative stress in patients with diabetic nephropathy, Nakamura 195-201 anthropometric indicators anthropometric indicators of obesity for identifying cardiometabolic risk factors in a rural Bangladeshi population, Bhowmik 361-368

anti-cancer ascorbic acid therapy

pseudohypoglycemia or hyperglycemia caused by interference with self-monitoring blood glucose measurements in anti-cancer ascorbic acid therapy (Letter to the Editor), Kimura 682

antidiabetic agents

new therapeutic strategy for Alzheimer's disease using antidiabetes agents (Commentary), Yokono 152-153

antioxidant

mechanism-based antioxidant therapies promise to prevent diabetic complications (Editorial), Nishikawa 105-107

arterial stiffness

increased arterial stiffness is closely associated with hyperglycemia and improved by glycemic control in diabetic patients, lbata $82-87$

Asian

changing characteristics of the type 2 diabetes epidemic in China and other Asian countries, Yang 223-224

efficacy and safety of exenatide once-weekly vs exenatide twice-daily in Asian patients with type 2 diabetes mellitus, Ji $\quad 53-61$

insulin degludec compared with insulin glargine in insulin-naïve patients with type 2 diabetes: a 26-week, randomized, controlled, Pan-Asian, treat-to-target trial, Onishi 605

atherosclerosis

extension of the mitochondria dysfunction hypothesis of metabolic syndrome to atherosclerosis with emphasis on the endocrine-disrupting chemicals and biophysical laws (Review Article), Lee 19-33

atorvastatin

comparison of effects of pitavastatin and atorvastatin on glucose metabolism in type 2 diabetic patients with hypercholesterolemia, Mita 297-303

effects of patient-tailored atorvastatin therapy on ameliorating the levels of atherogenic lipids and inflammation beyond lowering low-density lipoprotein cholesterol in patients with type 2 diabetes, Son $\quad 466-474$

azelnidipine

comparison of effects of cilnidipine and azelnidipine on blood pressure, heart rate and albuminuria in type 2 diabetics with hypertension: a pilot study, Abe 202-205

basal insulin

in search of the ideal basla insulin: does the new-generation ultra-long-acting insulin, dgludec, provide the answer? (Commentary), Hui 39-41

bleeding on probing

effect of glycemic control on periodontitis in type 2 diabetic patients with periodontal disease, Katagiri 320-325 
blood glucose

safety of exenatide once weekly for 52 weeks in Japanese patients with type 2 diabetes mellitus, Onishi 182-189

blood pressure variability

beneficial effect of calcium channel blockers on home blood pressure variability in the morning in patients with type 2 diabetes, Ushigome 399-404

body mass index

a 20-year trend of increasing obesity in young patients with poorly controlled type 2 diabetes at first diagnosis in urban Japan, Kushiyama 540

calcium channel blockers

beneficial effect of calcium channel blockers on home blood pressure variability in the morning in patients with type 2 diabetes, Ushigome 399-404

comparison of effects of cilnidipine and azelnidipine on blood pressure, heart rate and albuminuria in type 2 diabetics with hypertension: a pilot study, Abe 202-205

cancer

latest insights into the risk of cancer in diabetes (Review Article), Noto 225-232

cardiac autonomic neuropathy

diabetic cardiac autonomic neuropathy, inflammation and cardiovascular disease (Review Article), Vinik 4-18

cardio-ankle vascular index

increased arterial stiffness is closely associated with hyperglycemia and improved by glycemic control in diabetic patients, lbata 82-87

cardiovascular disease

diabetes mellitus, but not small dense low-density lipoprotein, is predictive of cardiovascular disease: A Korean community-based prospective cohort study, Suh 546

diabetic cardiac autonomic neuropathy, inflammation and cardiovascular disease (Review Article), Vinik 4-18

risk-benfit analysis of use of statins for primary prevention of cardiovascular disease in subjects without diabetes (Commentary), Chan 344-346

carnitine acyltransferase

metabolic flexibility and carnitine flux: the role of carnitine acyltransferase in glucose homeostasis (Commentary), Miyata 247-249

carotid plaque

association of glycated albumin with the presence of carotid plaque in patients with type 2 diabetes, Sato 638

$\beta$-cells

characterization of age-associated alterations of islet function and structure in diabetic mutant cryptochrome 1 transgenic mice, Okano 428-435

current status of regeneration of pancreatic $\beta$-cells (Review Article), Minami 131-141

new factors secreted from islets expand $\beta$-cell mass (Commentary), Watada 347-348

function

retrospective analysis of safety and efficacy of

insulin-to-liraglutide switch in Japanese type 2 diabetes: a caution against inappropriate use in patients with reduced $\beta$-cell function, Usui 585
China

changing characteristics of the type 2 diabetes epidemic in China and other Asian countries, Yang 223-224

chromium

glucagon and insulin have opposite effects on tissue chromium distribution in an obese mouse model, Lin 528

cigarette smoking

impact of cigarette smoking on impaired insulin secretion and insulin resistance in Japanese men: the Saku study,

Morimoto 274-280

cilnidipine

comparison of effects of cilnidipine and azelnidipine on blood pressure, heart rate and albuminuria in type 2 diabetics with hypertension: a pilot study, Abe 202-205

cohort study

family history of diabetes, lifestyle factors, and the 7-year incident risk of type 2 diabetes mellitus in middle-aged Japanese men and women, Sakurai 261-268

combination therapy

combination therapy with an angiotensin-converting-enzyme inhibitor and an angiotensin $\|$ receptor antagonist ameliorates microinflammation and oxidative stress in patients with diabetic nephropathy, Nakamura 195-201

Commentary

commentary on the effects of gestational hypertension on new-onset diabetes mellitus, Wang 245-246

FoxO1 breaks diabetic heart, Nagao 37-38

GATA transcription factors: new key regulators in pancreas organogenesis, Minami 426-427

glypican-4 is a new comer of adipokines working as insulin sensitizer, Tamori 250-251

in search of the ideal basla insulin: does the new-generation ultra-long-acting insulin, degludec, provide the answer?, Hui $\quad 39-41$ insulin resistance in the brain: a new therapeutic target for Alzheimer's disease, Umegaki 150-151

metabolic flexibility and carnitine flux: the role of carnitine acyltransferase in glucose homeostasis, Miyata 247-249 microalbuminuria and diabetic retinopathy in type 2 diabetic patients: from risk association to risk prediction, Hsuin 42-44 new factors secreted from islets expand $\beta$-cell mass, Watada 347-348 new therapeutic strategy for Alzheimer's disease using antidiabetes agents, Yokono 152-153

risk-benfit analysis of use of statins for primary prevention of cardiovascular disease in subjects without diabetes, Chan 344-346 sirtuin 1 as a key player of 'metabolic memory', Hashiramoto 34-36

VEGF-B as a therapeutic target to prevent ectopic fat deposition, Hosooka 525

what is the ideal biological marker in diagnosis of diabetic neuropathies?, Jin 154-156

complication

three ileus cases associated with the use of DPP-4 inhibitors in diabetic patients (Case Report), Kanasaki 676

confocal microscopy

morphometric features of corneal epithelial basal cells, and their relationship with corneal nerve pathology and clinical factors in patients with type 2 diabetes, Ishibashi 492-501 
continuous glucose monitoring

efficacy of $\alpha$-glucosidase inhibitors combined with dipeptidyl-peptidase-4 inhibitor (alogliptin) for glucose fluctuation in patients with type 2 diabetes mellitus by continuous glucose monitoring, Kurozumi 393-398

corneal epithelial basal cell morphometric features of corneal epithelial basal cells, and their relationship with corneal nerve pathology and clinical factors in patients with type 2 diabetes, Ishibashi 492-501

coronary disease

serum adiponectin levels predict the risk of coronary heart disease in Japanese patients with type 2 diabetes, Obata 475-482

C-peptide

identification and comparison of insulin pharmacokinetics injected with a new 4-mm needle vs 6- and 8-mm needles accounting for endogenous insulin and C-peptide secretion kinetics in non-diabetic adult males, Hirose 287-296

postprandial serum C-peptide value is the optimal index to identify patients with non-obese type 2 diabetes who require multiple daily insulin injection: analysis of C-peptide values before and after short-term intensive insulin therapy, Fujiwara 622

degludec

in search of the ideal basal insulin: does the new-generation ultra-long-acting insulin, dgludec, provide the answer? (Commentary), Hui 39-41

insulin degludec compared with insulin glargine in insulin-näve patients with type 2 diabetes: a 26-week, randomized, controlled, Pan-Asian, treat-to-target trial, Onishi 605

insulin degludec in Japanese patients with type 1 diabetes mellitus: a randomized controlled trial, Iwamoto 62-68

dementia

diabetes mellitus and risk of dementia: a meta-analysis of prospective observational studies, Kapil 644

diabetes

anti-inflammatory effects of exendin-4, a glucagon-like peptide-1 analog, on human peripheral lymphocytes in patients with type 2 diabetes, He 382-392

association between diabetes or antidiabetic therapy and lung cancer: a meta-analysis, Wang 662

care

diabetes care: after the Great East Japan Earthquake (Special

Report), Kishimoto 97-102

complications

glucose-dependent insulinotropic polypeptide and glucagon-like peptide-1: incretin actions beyond the pancreas (Review Article), Seino 108-130

mechanism-based antioxidant therapies promise to prevent diabetic complications (Editorial), Nishikawa 105-107 current status of regeneration of pancreatic $\beta$-cells (Review Article), Minami 131-141

duration

research on the relationships between pancreatic cancer and hyperglycemia in Chinese populations, Huang 45-52

effect of periodontal treatment on glycemic control of patients with diabetes: a systematic review and meta-analysis, Corbella 502-509 effects of walking on medical cost: a quantitative evaluation by simulation focusing on diabetes, Kato 675

latest insights into the risk of cancer in diabetes (Review Article), Noto 225-232

management

are information technology-based systems the way forward

for diabetes management in Asia? (Editorial), Cho 1-3

pharmacokinetics, pharmacodynamics, safety and tolerability of four weeks' treatment with empagliflozin in Japanese patients with type 2 diabetes mellitus, Kanada 613

see also type 1 diabetes

see also type 2 diabetes

diabetes mellitus

diabetes mellitus and risk of dementia: a meta-analysis of prospective observational studies, Kapil 644

increasing achievement of the target goals for glycemic, blood pressure and lipid control for adults with diagnosed diabetes in Korea, Yu 460-465

diabetic kidney disease

comparison of spironolactone and trichlormethiazide as add-on therapy to renin-angiotensin blockade for reduction of albuminuria in diabetic patients, Hase 316-319

diabetic nephropathy

combination therapy with an angiotensin-converting-enzyme inhibitor and an angiotensin II receptor antagonist ameliorates microinflammation and oxidative stress in patients with diabetic nephropathy, Nakamura 195-201

microinflammation in the pathogenesis of diabetic nephropathy (Review Article), Shikata 142

significance of renal biopsy in patients with presumed diabetic nephropathy, Harada 88-93

validity of the medical outcomes study sleep scale in patients with painful diabetic peripheral neuropathy in Korea, Kim 405-409

what is the ideal biological marker in diagnosis of diabetic neuropathies? (Commentary), Jin 154-156

diabetic retinopathy

angiotensin-converting enzyme gene variants interact with the renin-angiotensin system pathway to confer risk and protection against type 2 diabetic retinopathy (Letter to the Editor), Singh Cheema 103-104

microalbuminuria and diabetic retinopathy in type 2 diabetic patients: from risk association to risk prediction (Commentary), Hsuin 42-44

diagnostic rate significance of renal biopsy in patients with presumed diabetic nephropathy, Harada 88-93

differentiation

inhibition of preadipocyte differentiation and adipogenesis by zinc- $\alpha 2$-glycoprotein treatment in 3T3-L1 cells, Zhu 252-260

dipeptidyl peptidase-4 inhibitors

dipeptidyl peptidase-4 inhibitors are effective in Japanese type 2 diabetic patients with sustained endogenous insulin-secreting capacity, a higher body mass index and insulin resistance, Kozawa 190-194

efficacy and safety of teneligliptin in combination with pioglitazone in Japanese patients with type 2 diabetes mellitus, Kadowaki 576 
efficacy of $\boldsymbol{\alpha}$-glucosidase inhibitors combined with dipeptidyl-peptidase-4 inhibitor (alogliptin) for glucose fluctuation in patients with type 2 diabetes mellitus by continuous glucose monitoring, Kurozumi 393-398

three ileus cases associated with the use of DPP-4 inhibitors in diabetic patients (Case Report), Kanasaki 676

disasters

diabetes care: after the Great East Japan Earthquake (Special Report), Kishimoto 97-102

Editorial

are information technology-based systems the way forward for diabetes management in Asia?, Cho 1-3

changing characteristics of the type 2 diabetes epidemic in China and other Asian countries, Yang 223-224

insulin-dependent and -independent regulation of sterol regulatory element-binding protein 1c, Matsuzaka 411-412

mechanism-based antioxidant therapies promise to prevent diabetic complications, Nishikawa 105-107

searching for stem cells in the adult pancreas: a futile effort? Minami 331-333

empagliflozin

pharmacokinetics, pharmacodynamics, safety and tolerability of four weeks' treatment with empagliflozin in Japanese patients with type 2 diabetes mellitus, Kanada 613

endocrine-disrupting chemicals

extension of the mitochondria dysfunction hypothesis of metabolic syndrome to atherosclerosis with emphasis on the endocrine-disrupting chemicals and biophysical laws (Review Article), Lee 19-33

epidemiology

family history of diabetes, lifestyle factors, and the 7-year incident risk of type 2 diabetes mellitus in middle-aged Japanese men and women, Sakurai 261-268

exenatide

efficacy and safety of exenatide once-weekly vs exenatide twice-daily in Asian patients with type 2 diabetes mellitus, Ji 53-61

safety of exenatide once weekly for 52 weeks in Japanese patients with type 2 diabetes mellitus, Onishi 182-189

exendin-4

anti-inflammatory effects of exendin-4, a glucagon-like peptide-1 analog, on human peripheral lymphocytes in patients with type 2 diabetes, He 382-392

family history

family history of diabetes, lifestyle factors, and the 7-year incident risk of type 2 diabetes mellitus in middle-aged Japanese men and women, Sakurai 261-268

fasting plasma glucose

prevalence of retinopathy and its risk factors in a Japanese population, Fukushima 349-354

fat

VEGF-B as a therapeutic target to prevent ectopic fat deposition, Hosooka 525 fiber atrophy

impaired nerve fiber regeneration in axotomized peripheral nerve in streptozotocin-diabetic rats, Nishida 533

fibroblast growth factor-21

obesity as the common soil of non-alcoholic fatty liver disease and diabetes: role of adipokines (Review Article), Hui 413-425

flow-mediated vascular dilatation

combined examination of glyceryl trinitrate-mediated vascular dilation with flow-mediated vascular dilation is essential for assessment of vascular function in type 2 diabetes, Furuta 304-309

FoxO1

FoxO1 breaks diabetic heart (Commentary), Nagao 37-38

GATA transcription factors

GATA transcription factors: new key regulators in pancreas organogenesis, Minami 426-427

genetic association studies

replication study for the association of rs391300 in SRR and rs17584499 in PTPRD with susceptibility to type 2 diabetes in a Japanese population, Imamura 168-173

genetic mutations

transgenic zebrafish model of the C43G human insulin gene mutation, Eames 157-167

genetics

characterization of age-associated alterations of islet function and structure in diabetic mutant cryptochrome 1 transgenic mice, Okano 428-435

clinical and functional characterization of the Pro1198Leu ABCC8 gene mutation associated with permanent neonatal diabetes mellitus, Takagi 269-273

insights into the genetic basis of type 2 diabetes (Review Article), Kato 233-244

Kir6.2 E23K polymorphism is related to secondary failure of sulfonylureas in non-obese patients with type 2 diabetes, Shimajiri 445-449

protective role of human insulin against the cytotoxicity associated with human mutant S20G islet amyloid polypeptide, Morita 436- 444

gestational hypertenstion

commentary on the effects of gestational hypertension on new-onset diabetes mellitus (Commentary), Wang 245-246 glucagon

glucagon and insulin have opposite effects on tissue chromium distribution in an obese mouse model, Lin 528

glucagon-like peptide-1

anti-inflammatory effects of exendin-4, a glucagon-like peptide-1 analog, on human peripheral lymphocytes in patients with type 2 diabetes, He 382-392

glucose-dependent insulinotropic polypeptide and glucagon-like peptide-1: incretin actions beyond the pancreas (Review Article), Seino 108-130

liraglutide administration in type 2 diabetic patients who either received no previous treatment or were treated with an oral hypoglycemic agent showed greater efficacy than that in patients switching from insulin, Nambu 69-77 
glucagon-like peptide-1 receptor agonist

retrospective analysis of safety and efficacy of insulin-to-liraglutide switch in Japanese type 2 diabetes: a caution against inappropriate use in patients with reduced $\beta$-cell function, Usui 585

glucagon stimulation test

defining criteria for the introduction of liraglutide using the glucagon stimulation test in patients with type 2 diabetes, Kondo 571

retrospective analysis of safety and efficacy of insulin-to-liraglutide switch in Japanese type 2 diabetes: a caution against inappropriate use in patients with reduced $\beta$-cell function, Usui 585

glucose area under the curve

usefulness of a novel system for measuring glucose area under the curve while screening for glucose intolerance in outpatients, Sakamoto 552

glucose homeostasis

metabolic flexibility and carnitine flux: the role of carnitine acyltransferase in glucose homeostasis (Commentary), Miyata 247-249

glucose metabolism

comparison of effects of pitavastatin and atorvastatin on glucose metabolism in type 2 diabetic patients with hypercholesterolemia, Mita 297-303

glucose monitoring

usefulness of a novel system for measuring glucose area under the curve while screening for glucose intolerance in outpatients, Sakamoto 552

glucose-dependent insulinotropic polypeptide glucose-dependent insulinotropic polypeptide and glucagon-like peptide-1: incretin actions beyond the pancreas (Review Article), Seino 108-130

$\alpha$-glucosidase inhibitor efficacy of $\alpha$-glucosidase inhibitors combined with dipeptidyl-peptidase-4 inhibitor (alogliptin) for glucose fluctuation in patients with type 2 diabetes mellitus by continuous glucose monitoring, Kurozumi 393-398

glycated albumin

a 20-year trend of increasing obesity in young patients with poorly controlled type 2 diabetes at first diagnosis in urban Japan, Kushiyama 540

association of glycated albumin with the presence of carotid plaque in patients with type 2 diabetes, Sato 638

effects of insulin changes on quality of life and glycaemic control in Japanese patients with type 2 diabetes mellitus: the INSIGHTs observational study, Ishii 560

morningness-eveningness questionnaire score correlates with glycated hemoglobin in middle-aged male workers with type 2 diabetes mellitus, Iwasaki 376-381

prevalence of retinopathy and its risk factors in a Japanese population, Fukushima 349-354

self-perception of glycemic control among Japanese type 2 diabetic patients: accuracy of patient perception and characteristics of patients with misperception, Suzuki-Saito 206-213 glycemic control

addition of sitagliptin to ongoing metformin monotherapy improves glycemic control in Japanese patients with type 2 diabetes over 52 weeks, Kadowaki 174-181

effect of glycemic control on periodontitis in type 2 diabetic patients with periodontal disease, Katagiri 320-325

increased arterial stiffness is closely associated with hyperglycemia and improved by glycemic control in diabetic patients, Ibata $82-87$

increasing achievement of the target goals for glycemic, blood pressure and lipid control for adults with diagnosed diabetes in Korea, Yu 460-465

self-perception of glycemic control among Japanese type 2 diabetic patients: accuracy of patient perception and characteristics of patients with misperception, Suzuki-Saito 206-213

structured self-monitoring of blood glucose reduces glycated hemoglobin in insulin-treated diabetes, Kato 450-453 weight reduction is associated with improvement of glycemic control in Japanese men, whose hemoglobin A1C is 5.6-6.4\%, with visceral fat accumulation, but not without visceral fat accumulation, Okauchi 454-459

glyceryl trinitrate-mediated vascular dilatation

combined examination of glyceryl trinitrate-mediated vascular dilation with flow-mediated vascular dilation is essential for assessment of vascular function in type 2 diabetes, Furuta 304-309

glycosylated hemoglobin A increasing achievement of the target goals for glycemic, blood pressure and lipid control for adults with diagnosed diabetes in Korea, Yu 460-465

glypican-4

glypican-4 is a new comer of adipokines working as insulin sensitizer (Commentary), Tamori 250-251

Great East Japan Earthquake diabetes care: after the Great East Japan Earthquake (Special Report), Kishimoto 97-102

HbA1c see glycosylated hemoglobin

home blood pressure monitoring

beneficial effect of calcium channel blockers on home blood pressure variability in the morning in patients with type 2 diabetes, Ushigome 399-404

3-hydroxy-3-methylglutaryl coenzyme A reductase inhibitor comparison of effects of pitavastatin and atorvastatin on glucose metabolism in type 2 diabetic patients with hypercholesterolemia, Mita 297-303

hypercholesterolemia comparison of effects of pitavastatin and atorvastatin on glucose metabolism in type 2 diabetic patients with hypercholesterolemia, Mita 297-303

hyperglycemia

increased arterial stiffness is closely associated with hyperglycemia and improved by glycemic control in diabetic patients, Ibata 82-87

pseudohypoglycemia or hyperglycemia caused by interference with self-monitoring blood glucose measurements in anti-cancer ascorbic acid therapy (Letter to the Editor), Kimura 682 
research on the relationships between pancreatic cancer and hyperglycemia in Chinese populations, Huang 45-52

hypertension

comparison of effects of cilnidipine and azelnidipine on blood pressure, heart rate and albuminuria in type 2 diabetics with hypertension: a pilot study, Abe 202-205

see also gestational hypertension

hypoglycemia

regular insulin, rather than rapid-acting insulin, is a suitable choice for premeal bolus insulin in lean patients with type 2 diabetes mellitus, Kuroda 78-81

impaired insulin secretion

impact of cigarette smoking on impaired insulin secretion and insulin resistance in Japanese men: the Saku study, Morimoto 274-280

incretin

effects of the naturally-occurring disaccharides, palatinose and sucrose, on incretin secretion in healthy non-obese subjects, Maeda 281-286

glucose-dependent insulinotropic polypeptide and glucagon-like peptide-1: incretin actions beyond the pancreas (Review Article), Seino 108-130

liraglutide administration in type 2 diabetic patients who either received no previous treatment or were treated with an oral hypoglycemic agent showed greater efficacy than that in patients switching from insulin, Nambu 69-77

sitagliptin added to voglibose monotherapy improves glycemic control in patients with type 2 diabetes, Tajima 595

inflammation

anti-inflammatory effects of exendin-4, a glucagon-like peptide-1 analog, on human peripheral lymphocytes in patients with type 2 diabetes, He 382-392

diabetic cardiac autonomic neuropathy, inflammation and cardiovascular disease (Review Article), Vinik 4-18

effects of patient-tailored atorvastatin therapy on ameliorating the levels of atherogenic lipids and inflammation beyond lowering low-density lipoprotein cholesterol in patients with type 2 diabetes, Son $\quad 466-474$

microinflammation in the pathogenesis of diabetic nephropathy (Review Article), Shikata 142

information technology

are information technology-based systems the way forward for diabetes management in Asia? (Editorial), Cho 1-3 insulin

current status of regeneration of pancreatic $\beta$-cells (Review Article), Minami 131-141

glucagon and insulin have opposite effects on tissue chromium distribution in an obese mouse model, Lin 528 identification and comparison of insulin pharmacokinetics injected with a new 4-mm needle vs 6- and 8-mm needles accounting for endogenous insulin and C-peptide secretion kinetics in non-diabetic adult males, Hirose 287-296

insulin-dependent and -independent regulation of sterol regulatory element-binding protein 1c (Editorial), Matsuzaka $411-412$ modification of a simple clinical scoring system as a diagnostic screening tool for nonalcoholic steatohepatitis in Japanese patients with nonalcoholic fatty liver disease, Nakamura 655 protective role of human insulin against the cytotoxicity associated with human mutant S20G islet amyloid polypeptide, Morita 436444

regular insulin, rather than rapid-acting insulin, is a suitable choice for premeal bolus insulin in lean patients with type 2 diabetes mellitus, Kuroda 78-81

insulin allergy

successful control of a case of severe insulin allergy with liraglutide, Kawanami 94-96

insulin analogs and derivatives

insulin degludec in Japanese patients with type 1 diabetes mellitus: a randomized controlled trial, Iwamoto $62-68$

insulin gene

transgenic zebrafish model of the C43G human insulin gene mutation, Eames 157-167

insulin resistance

dipeptidyl peptidase-4 inhibitors are effective in Japanese type 2 diabetic patients with sustained endogenous insulin-secreting capacity, a higher body mass index and insulin resistance, Kozawa 190-194

distribution of the Matsuda index in Japanese healthy subjects, Takahara 369-371

impact of cigarette smoking on impaired insulin secretion and insulin resistance in Japanese men: the Saku study,

Morimoto 274-280

insulin resistance in the brain: a new therapeutic target for Alzheimer's disease (Commentary), Umegaki 150-151

Role of thiazolidinediones, insulin sensitizer in nonalcoholic fatty liver disease (NAFLD) (Review Article), Chang 517

insulin secretion

characterization of age-associated alterations of islet function and structure in diabetic mutant cryptochrome 1 transgenic mice, Okano 428-435

dipeptidyl peptidase-4 inhibitors are effective in Japanese type 2 diabetic patients with sustained endogenous insulin-secreting capacity, a higher body mass index and insulin resistance, Kozawa 190-194

physiological glucose-stimulated insulin secretion: a newer perspective (Review Article), Komatsu 511

insulin sensitizer

glypican-4 is a new comer of adipokines working as insulin sensitizer, Tamori 250-251

insulin therapy-related quality of life questionnaire

effects of insulin changes on quality of life and glycaemic control in Japanese patients with type 2 diabetes mellitus: the INSIGHTS observational study, Ishii 560

insulin-treated diabetes

structured self-monitoring of blood glucose reduces glycated hemoglobin in insulin-treated diabetes, Kato 450-453

irregular menstrual cycles

ovarian morphology and prevalence of polycystic ovary syndrome in Japanese women with type 1 diabetes mellitus, Miyoshi 326-329 
ischemic heart disease

ischemic heart disease is associated with vertebral fractures in patients with type 2 diabetes mellitus, Muñoz-Torres 310-315

islet amyloid polypeptide

protective role of human insulin against the cytotoxicity associated with human mutant S20G islet amyloid polypeptide, Morita 436-444

islet architecture

characterization of age-associated alterations of islet function and structure in diabetic mutant cryptochrome 1 transgenic mice, Okano 428-435

islets of Langerhans

transgenic zebrafish model of the C43G human insulin gene mutation, Eames 157-167

Japanese

meaning of upper limit of normal range of post-load 1-h plasma glucose level defined by oral glucose tolerance test in Japanese subjects, Iwao 372-375

morningness-eveningness questionnaire score correlates with glycated hemoglobin in middle-aged male workers with type 2 diabetes mellitus, Iwasaki 376-381

prevalence of retinopathy and its risk factors in a Japanese population, Fukushima 349-354

replication study for the association of rs391300 in SRR and rs17584499 in PTPRD with susceptibility to type 2 diabetes in a Japanese population, Imamura 168-173

safety of exenatide once weekly for 52 weeks in Japanese patients with type 2 diabetes mellitus, Onishi 182-189

KATP channel

glucose-stimulated insulin secretion: a newer perspective (Review Article), Komatsu 511

Kir6.2 E23K polymorphism

Kir6.2 E23K polymorphism is related to secondary failure of sulfonylureas in non-obese patients with type 2 diabetes, Shimajiri 445-449

Kyoto Declaration on Diabetes

promoting research for better diabetes care in Asia, Asian Association for the Study of Diabetes 222

Letter to the Editor

angiotensin-converting enzyme gene variants interact with the renin-angiotensin system pathway to confer risk and protection against type 2 diabetic retinopathy, Singh Cheema 103-104

pseudohypoglycemia or hyperglycemia caused by interference with self-monitoring blood glucose measurements in anti-cancer ascorbic acid therapy, Kimura 682

liraglutide

defining criteria for the introduction of liraglutide using the glucagon stimulation test in patients with type 2 diabetes, Kondo 571

liraglutide administration in type 2 diabetic patients who either received no previous treatment or were treated with an oral hypoglycemic agent showed greater efficacy than that in patients switching from insulin, Nambu 69-77 successful control of a case of severe insulin allergy with liraglutide, Kawanami 94-96

long-acting insulin insulin degludec in Japanese patients with type 1 diabetes mellitus: a randomized controlled trial, Iwamoto $62-68$

low-density lipoprotein cholesterol

effects of patient-tailored atorvastatin therapy on ameliorating the levels of atherogenic lipids and inflammation beyond lowering low-density lipoprotein cholesterol in patients with type 2 diabetes, Son $\quad 466-474$

low-density lipoprotein particle size diabetes mellitus, but not small dense low-density lipoprotein, is predictive of cardiovascular disease: A Korean community-based prospective cohort study, Suh 546

lung cancer association between diabetes or antidiabetic therapy and lung cancer: a meta-analysis, Wang 662

Matsuda index distribution of the Matsuda index in Japanese healthy subjects, Takahara 369-371

meal load

postprandial serum C-peptide value is the optimal index to identify patients with non-obese type 2 diabetes who require multiple daily insulin injection: analysis of C-peptide values before and after short-term intensive insulin therapy, Fujiwara 622

medical costs

effects of walking on medical cost: a quantitative evaluation by simulation focusing on diabetes, Kato 675

meta-analysis association between diabetes or antidiabetic therapy and lung cancer: a meta-analysis, Wang 662

diabetes mellitus and risk of dementia: a meta-analysis of prospective observational studies, Kapil 644

metabolic control effect of periodontal treatment on glycemic control of patients with diabetes: a systematic review and meta-analysis, Corbella 502-509

metabolic memory

sirtuin 1 as a key player of 'metabolic memory' (Commentary), Hashiramoto 34-36

metabolic syndrome extension of the mitochondria dysfunction hypothesis of metabolic syndrome to atherosclerosis with emphasis on the endocrine-disrupting chemicals and biophysical laws (Review Article), Lee 19-33

metabolic syndrome as a predictor of type 2 diabetes, and its clinical interpretations and usefulness (Review Article), Shin 334-343

metformin

addition of sitagliptin to ongoing metformin monotherapy improves glycemic control in Japanese patients with type 2 diabetes over 52 weeks, Kadowaki 174-181

microalbuminuria

microalbuminuria and diabetic retinopathy in type 2 diabetic patients: from risk association to risk prediction (Commentary), Hsuin 42-44 
microinflammation

combination therapy with an angiotensin-converting-enzyme inhibitor and an angiotensin II receptor antagonist ameliorates microinflammation and oxidative stress in patients with diabetic nephropathy, Nakamura 195-201

microinflammation in the pathogenesis of diabetic nephropathy (Review Article), Shikata 142

misfolded proinsulin

transgenic zebrafish model of the C43G human insulin gene mutation, Eames 157-167

mitochondrial dysfunction

extension of the mitochondria dysfunction hypothesis of metabolic syndrome to atherosclerosis with emphasis on the endocrine-disrupting chemicals and biophysical laws (Review Article), Lee 19-33

modulatory signals

glucose-stimulated insulin secretion: a newer perspective (Review

Article), Komatsu 511

morningness-eveningness questionnaire

morningness-eveningness questionnaire score correlates with glycated hemoglobin in middle-aged male workers with type 2 diabetes mellitus, Iwasaki 376-381

multiple daily insulin injections

postprandial serum C-peptide value is the optimal index to identify patients with non-obese type 2 diabetes who require multiple daily insulin injection: analysis of C-peptide values before and after short-term intensive insulin therapy, Fujiwara 622

myelination

impaired nerve fiber regeneration in axotomized peripheral nerve in streptozotocin-diabetic rats, Nishida 533

NAFLD see nonalcoholic fatty liver disease

needle length

identification and comparison of insulin pharmacokinetics injected with a new 4-mm needle vs 6- and 8-mm needles accounting for endogenous insulin and C-peptide secretion kinetics in non-diabetic adult males, Hirose 287-296

neonatal diabetes

clinical and functional characterization of the Pro1198Leu ABCC8 gene mutation associated with permanent neonatal diabetes mellitus, Takagi 269-273

transgenic zebrafish model of the C43G human insulin gene mutation, Eames 157-167

nerve regeneration

impaired nerve fiber regeneration in axotomized peripheral nerve in streptozotocin-diabetic rats, Nishida 533

niveau

three ileus cases associated with the use of DPP-4 inhibitors in diabetic patients (Case Report), Kanasaki 676

nonalcoholic fatty liver disease

modification of a simple clinical scoring system as a diagnostic screening tool for nonalcoholic steatohepatitis in Japanese patients with nonalcoholic fatty liver disease, Nakamura 655

obesity as the common soil of non-alcoholic fatty liver disease and diabetes: role of adipokines (Review Article), Hui 413-425 role of thiazolidinediones, insulin sensitizer in nonalcoholic fatty liver disease (NAFLD) (Review Article), Chang 517

obesity

anthropometric indicators of obesity for identifying cardiometabolic risk factors in a rural Bangladeshi population, Bhowmik 361-368 obesity as the common soil of non-alcoholic fatty liver disease and diabetes: role of adipokines (Review Article), Hui 413-425

oral glucose tolerance test

meaning of upper limit of normal range of post-load 1-h plasma glucose level defined by oral glucose tolerance test in Japanese subjects, Iwao 372-375

oxidative stress

combination therapy with an angiotensin-converting-enzyme inhibitor and an angiotensin $\|$ receptor antagonist ameliorates microinflammation and oxidative stress in patients with diabetic nephropathy, Nakamura 195-201

pain

validity of the medical outcomes study sleep scale in patients with painful diabetic peripheral neuropathy in Korea, Kim 405-409

palatinose

effects of the naturally-occurring disaccharides, palatinose and sucrose, on incretin secretion in healthy non-obese subjects, Maeda 281-286

pancreas

GATA transcription factors: new key regulators in pancreas organogenesis, Minami 426-427

pancreatic cancer

research on the relationships between pancreatic cancer and hyperglycemia in Chinese populations, Huang 45-52

pathogenesis

diabetic cardiac autonomic neuropathy, inflammation and cardiovascular disease (Review Article), Vinik 4-18

pathology

transgenic zebrafish model of the C43G human insulin gene mutation, Eames 157-167

periodontal disease

effect of periodontal treatment on glycemic control of patients with diabetes: a systematic review and meta-analysis, Corbella 502-509

periodontitis

effect of glycemic control on periodontitis in type 2 diabetic patients with periodontal disease, Katagiri 320-325

pharmacokinetics

identification and comparison of insulin pharmacokinetics injected with a new 4-mm needle vs 6- and 8-mm needles accounting for endogenous insulin and C-peptide secretion kinetics in non-diabetic adult males, Hirose 287-296

pioglitazone

efficacy and safety of teneligliptin in combination with pioglitazone in Japanese patients with type 2 diabetes mellitus, Kadowaki 576 pitavastatin

comparison of effects of pitavastatin and atorvastatin on glucose metabolism in type 2 diabetic patients with hypercholesterolemia, Mita 297-303 
plasma glucose

insights into the genetic basis of type 2 diabetes (Review Article),

Kato 233-244

polycystic ovary syndrome

ovarian morphology and prevalence of polycystic ovary syndrome in Japanese women with type 1 diabetes mellitus, Miyoshi 326329

post-load hyperglycemia

effects of soybean product intake on fasting and postload hyperglycemia and type 2 diabetes in Japanese men with high BMI: The Saku Study, Tatsumi 630

post-load 1-h plasma glucose

meaning of upper limit of normal range of post-load 1-h plasma glucose level defined by oral glucose tolerance test in Japanese subjects, Iwao 372-375

prevalence

increasing achievement of the target goals for glycemic, blood pressure and lipid control for adults with diagnosed diabetes in Korea, Yu 460-465

pseudohypoglycemia

pseudohypoglycemia or hyperglycemia caused by interference with self-monitoring blood glucose measurements in anti-cancer ascorbic acid therapy (Letter to the Editor), Kimura 682

reference limit

distribution of the Matsuda index in Japanese healthy subjects,

Takahara 369-371

regenerative medicine

current status of regeneration of pancreatic $\beta$-cells (Review Article), Minami 131-141

regional pain syndrome

correlation of thalamic blood flow redistribution with persistent complex regional pain syndrome in a stroke patient with poor diabetic control (Case Report), Hsu 679

renal biopsy

significance of renal biopsy in patients with presumed diabetic nephropathy, Harada 88-93

renin-angiotensin system

angiotensin-converting enzyme gene variants interact with the renin-angiotensin system pathway to confer risk and protection against type 2 diabetic retinopathy (Letter to the Editor), Singh Cheema 103-104

combination therapy with an angiotensin-converting-enzyme inhibitor and an angiotensin II receptor antagonist ameliorates microinflammation and oxidative stress in patients with diabetic nephropathy, Nakamura 195-201

comparison of spironolactone and trichlormethiazide as add-on therapy to renin-angiotensin blockade for reduction of albuminuria in diabetic patients, Hase 316-319

retinopathy

prevalence of retinopathy and its risk factors in a Japanese population, Fukushima 349-354

risk factors

cardiometabolic

anthropometric indicators of obesity for identifying cardiometabolic risk factors in a rural Bangladeshi population, Bhowmik 361-368 latest insights into the risk of cancer in diabetes (Review Article),

Noto 225-232

metabolic syndrome as a predictor of type 2 diabetes, and its clinical interpretations and usefulness (Review Article), Shin 334 343

research on the relationships between pancreatic cancer and hyperglycemia in Chinese populations, Huang 45-52

S20G mutation

protective role of human insulin against the cytotoxicity associated with human mutant S20G islet amyloid polypeptide, Morita 444

screening

a 20-year trend of increasing obesity in young patients with poorly controlled type 2 diabetes at first diagnosis in urban Japan, Kushiyama 540

usefulness of a novel system for measuring glucose area under the curve while screening for glucose intolerance in outpatients,

Sakamoto 552

self-monitoring of blood glucose

pseudohypoglycemia or hyperglycemia caused by interference with self-monitoring blood glucose measurements in anti-cancer ascorbic acid therapy (Letter to the Editor), Kimura 682 structured self-monitoring of blood glucose reduces glycated hemoglobin in insulin-treated diabetes, Kato 450-453

self-perception

self-perception of glycemic control among Japanese type 2 diabetic patients: accuracy of patient perception and characteristics of patients with misperception, Suzuki-Saito 206-213

simulation

effects of walking on medical cost: a quantitative evaluation by simulation focusing on diabetes, Kato 675

sirtuin 1

sirtuin 1 as a key player of 'metabolic memory' (Commentary), Hashiramoto 34-36

sitagliptin

addition of sitagliptin to ongoing metformin monotherapy improves glycemic control in Japanese patients with type 2 diabetes over 52 weeks, Kadowaki 174-181

sitagliptin added to voglibose monotherapy improves glycemic control in patients with type 2 diabetes, Tajima 595 sleep

validity of the medical outcomes study sleep scale in patients with painful diabetic peripheral neuropathy in Korea, Kim 405-409

small dense low-density lipoprotein

diabetes mellitus, but not small dense low-density lipoprotein, is predictive of cardiovascular disease: A Korean community-based prospective cohort study, Suh 546

sodium glucose cotransporter 2 inhibitor

pharmacokinetics, pharmacodynamics, safety and tolerability of four weeks' treatment with empagliflozin in Japanese patients with type 2 diabetes mellitus, Kanada 613

soybean

effects of soybean product intake on fasting and postload hyperglycemia and type 2 diabetes in Japanese men with high BMl: The Saku Study, Tatsumi 630 
statins

risk-benfit analysis of use of statins for primary prevention of cardiovascular disease in subjects without diabetes (Commentary), Chan 344-346

stem cells

searching for stem cells in the adult pancreas: a futile effort?

(Editorial), Minami 331-333

sterol regulatory element-binding protein $1 \mathrm{c}$

insulin-dependent and -independent regulation of sterol regulatory element-binding protein 1c (Editorial), Matsuzaka 411-412

sucrose

effects of the naturally-occurring disaccharides, palatinose and sucrose, on incretin secretion in healthy non-obese subjects, Maeda 281-286

sulfonylurea receptor

clinical and functional characterization of the Pro1198Leu ABCC8

gene mutation associated with permanent neonatal diabetes mellitus, Takagi 269-273

sulfonylurea, secondary failure of

Kir6.2 E23K polymorphism is related to secondary failure of sulfonylureas in non-obese patients with type 2 diabetes, Shimajiri 445-449

3T3-L1 preadipocytes

inhibition of preadipocyte differentiation and adipogenesis by zinc- $\alpha 2$-glycoprotein treatment in 3T3-L1 cells, Zhu 252-260

thalamus

correlation of thalamic blood flow redistribution with persistent complex regional pain syndrome in a stroke patient with poor diabetic control (Case Report), Hsu 679

thiazide diuretics

comparison of spironolactone and trichlormethiazide as add-on therapy to renin-angiotensin blockade for reduction of albuminuria in diabetic patients, Hase 316-319

thiazolidinediones

Role of thiazolidinediones, insulin sensitizer in nonalcoholic fatty liver disease (NAFLD) (Review Article), Chang 517

type 1 diabetes

insulin degludec in Japanese patients with type 1 diabetes mellitus: a randomized controlled trial, Iwamoto 62-68

ovarian morphology and prevalence of polycystic ovary syndrome in Japanese women with type 1 diabetes mellitus, Miyoshi 326-329

type 2 diabetes

addition of sitagliptin to ongoing metformin monotherapy improves glycemic control in Japanese patients with type 2 diabetes over 52 weeks, Kadowaki 174-181

association of glycated albumin with the presence of carotid plaque in patients with type 2 diabetes, Sato 638

beneficial effect of calcium channel blockers on home blood pressure variability in the morning in patients with type 2 diabetes, Ushigome 399-404

changing characteristics of the type 2 diabetes epidemic in China and other Asian countries (Editorial), Yang 223-224 combined examination of glyceryl trinitrate-mediated vascular dilation with flow-mediated vascular dilation is essential for assessment of vascular function in type 2 diabetes, Furuta 304-309

comparison of effects of cilnidipine and azelnidipine on blood pressure, heart rate and albuminuria in type 2 diabetics with hypertension: a pilot study, Abe 202-205

comparison of effects of pitavastatin and atorvastatin on glucose metabolism in type 2 diabetic patients with hypercholesterolemia, Mita 297-303

correlation of thalamic blood flow redistribution with persistent complex regional pain syndrome in a stroke patient with poor diabetic control (Case Report), Hsu 679

defining criteria for the introduction of liraglutide using the glucagon stimulation test in patients with type 2 diabetes, Kondo 571

dipeptidyl peptidase-4 inhibitors are effective in Japanese type 2 diabetic patients with sustained endogenous insulin-secreting capacity, a higher body mass index and insulin resistance, Kozawa 190-194

effects of insulin changes on quality of life and glycaemic control in Japanese patients with type 2 diabetes mellitus: the INSIGHTs observational study, Ishii 560

effects of patient-tailored atorvastatin therapy on ameliorating the levels of atherogenic lipids and inflammation beyond lowering low-density lipoprotein cholesterol in patients with type 2 diabetes, Son $\quad 466-474$

effects of soybean product intake on fasting and postload hyperglycemia and type 2 diabetes in Japanese men with high BMl: The Saku Study, Tatsumi 630

effects of the naturally-occurring disaccharides, palatinose and sucrose, on incretin secretion in healthy non-obese subjects, Maeda 281-286

efficacy and safety of exenatide once-weekly vs exenatide twice-daily in Asian patients with type 2 diabetes mellitus, Ji 53-61

efficacy and safety of teneligliptin in combination with pioglitazone in Japanese patients with type 2 diabetes mellitus, Kadowaki 576 insights into the genetic basis of type 2 diabetes (Review Article), Kato 233-244

insulin degludec compared with insulin glargine in insulin-naive patients with type 2 diabetes: a 26-week, randomized, controlled, Pan-Asian, treat-to-target trial, Onishi 605

intervention therapy

effect of glycemic control on periodontitis in type 2 diabetic patients with periodontal disease, Katagiri 320-325

ischemic heart disease is associated with vertebral fractures in patients with type 2 diabetes mellitus, Muñoz-Torres 310-315 Kir6.2 E23K polymorphism is related to secondary failure of sulfonylureas in non-obese patients with type 2 diabetes, Shimajiri 445-449

liraglutide administration in type 2 diabetic patients who either received no previous treatment or were treated with an oral hypoglycemic agent showed greater efficacy than that in patients switching from insulin, Nambu 69-77 
metabolic syndrome as a predictor of type 2 diabetes, and its clinical interpretations and usefulness (Review Article), Shin 334-343

microalbuminuria and diabetic retinopathy in type 2 diabetic patients: from risk association to risk prediction (Commentary), Hsuin 42-44

morningness-eveningness questionnaire score correlates with glycated hemoglobin in middle-aged male workers with type 2 diabetes mellitus, Iwasaki 376-381

morphometric features of corneal epithelial basal cells, and their relationship with corneal nerve pathology and clinical factors in patients with type 2 diabetes, Ishibashi 492-501

regular insulin, rather than rapid-acting insulin, is a suitable choice for premeal bolus insulin in lean patients with type 2 diabetes mellitus, Kuroda 78-81

replication study for the association of rs391300 in SRR and rs 17584499 in PTPRD with susceptibility to type 2 diabetes in a Japanese population, Imamura 168-173

safety of exenatide once weekly for 52 weeks in Japanese patients with type 2 diabetes mellitus, Onishi 182-189

serum adiponectin levels predict the risk of coronary heart disease in Japanese patients with type 2 diabetes, Obata $475-482$

type B insulin resistance syndrome

successful control of a case of severe insulin allergy with liraglutide, Kawanami 94-96 vascular function

combined examination of glyceryl trinitrate-mediated vascular dilation with flow-mediated vascular dilation is essential for assessment of vascular function in type 2 diabetes, Furuta 304-309

VEGF-B

VEGF-B as a therapeutic target to prevent ectopic fat deposition, Hosooka 525

vertebral fractures

ischemic heart disease is associated with vertebral fractures in patients with type 2 diabetes mellitus, Muñoz-Torres 310-315

visceral fat accumulation

weight reduction is associated with improvement of glycemic control in Japanese men, whose hemoglobin A1C is 5.6-6.4\%, with visceral fat accumulation, but not without visceral fat accumulation, Okauchi $454-459$

voglibose

sitagliptin added to voglibose monotherapy improves glycemic control in patients with type 2 diabetes, Tajima 595

weight reduction

weight reduction is associated with improvement of glycemic control in Japanese men, whose hemoglobin A1C is 5.6-6.4\%, with visceral fat accumulation, but not without visceral fat accumulation, Okauchi 454-459

zinc- $\alpha 2$-glycoprotein inhibition of preadipocyte differentiation and adipogenesis by zinc- $\alpha 2$-glycoprotein treatment in 3T3-L1 cells, Zhu 252-260 